\title{
Model-Driven Development of Audio-Visual Web Search Applications: The PHAROS Demonstration
}

\author{
Alessandro Bozzon, Marco Brambilla, and Piero Fraternali \\ Politecnico di Milano, Piazza Leonardo Da Vinci, 32 - 20133 Milano, Italy \\ \{alessandro.bozzon, marco . brambilla, piero.fraternali\}@polimi.it
}

\begin{abstract}
PHAROS1 is an EU-founded project aimed at building a platform for advanced audiovisual search applications. In this demo we show the application of a Model-Driven Development (MDD) approach to the PHAROS demonstrator, which consists of an audio-visual Web search portal. The demo highlights the peculiar needs of search based applications and describes how existing MDD approaches can help fulfilling such needs, through visual modeling and automatic generation of RIA code for the front-end and business processes for the back-end.
\end{abstract}

\section{Introduction}

Due to the tremendous growth in the amount of digital data on the Web, search has become the default paradigm for interacting with contents. Multimedia search portals, which are now the access channels of choice, typically comprise two major flows of activities: 1) the Query and Result Presentation (QRP) process, which encompasses query preprocessing, query execution, and results post-processing, and 2) the Content Provisioning (CP) process, which gets content from its original location, analyzes (or annotates) it, and makes it available to the search engines for later search.

Depending on the targeted application domain, the QRP and CP processes have to be tailored to a wide spectrum of functional and non functional requirements, that put at stake the advantages of current Web Engineering approaches [5] [7.

Some typical challenges are related to the complexity deriving from: (i) the adopted query modality (e.g., keyword-based, similarity search, etc.), (ii) the required user interaction patterns (e.g., searching, monitoring, and browsing [2]), (iii) the presence of social interaction features (e.g., tagging), (iv) the need for flexible and distributable composition of annotation technologies (imposed by business or performance requirements), (v) highly competitive and quickly changing marketing strategies typical of the Web environment, etc. The design, development and integration of search engines systems therefore result in an articulated, complex task, involving different knowledge and skills. This demonstration will show that such needs might be fulfilled by adopting and extending

${ }^{1}$ http://www.pharos-audiovisual-search.eu 


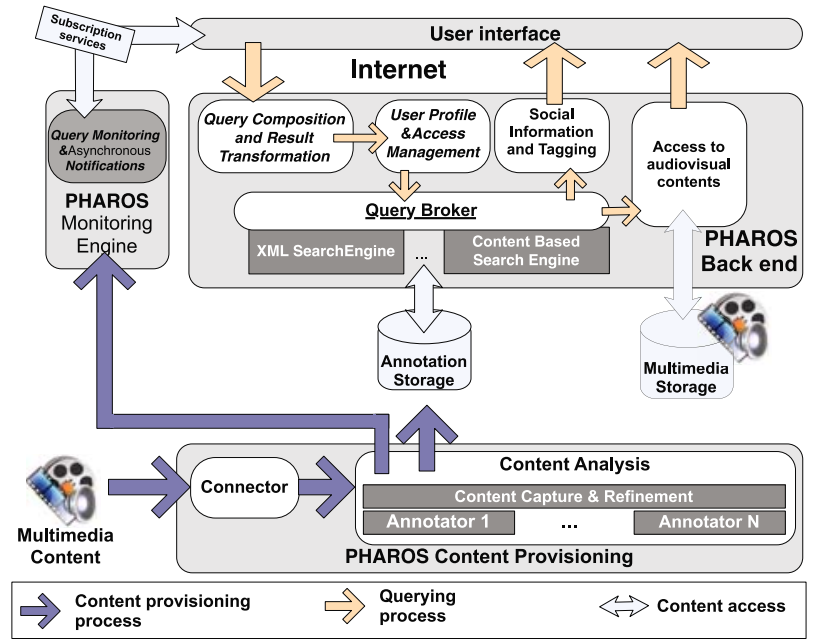

Fig. 1. A high-level view of the PHAROS platform architecture

the methods and tools that Web engineering provides for conceptual modeling of traditional Web applications.

\section{Demonstration Scenario}

Our work stems from the requirements gathered within PHAROS (Platform for searcHing of Audiovisual Resources across Online Spaces) [4, an Integrated Project funded by the European Union in the Sixth Framework Programme; its goal is to. Figure 1 depicts a high level view of the PHAROS platform architecture, comprising a set of components that interact according to the SOA paradigm for performing the $\mathrm{CP}$ and QRP and execution flows.

In PHAROS, contents are represented both by manual metadata (e.g, title and description) and annotations automatically generated by the PHAROS platform during the CP process (e.g speech-to-text transcriptions, speaker's gender or name, music mood and rhythm, etc.). Annotations refer to temporal segments of occurrence, which is exploited in the QRP process to enable navigation of videos according to the temporal segment of occurrence for a given query match. The PHAROS QRP process supports several combinations of user interaction patterns and query modalities, like content-based queries based on music, images and faces, browsing based on automatically generated annotations, search by user-generated tags, etc.

This demonstration includes: (i) the description of the MDD approach adopted for the design of the QRP process, based on the WebML notation, that allow to produce rich web interfaces; (ii) the description of the MDD approach for the 


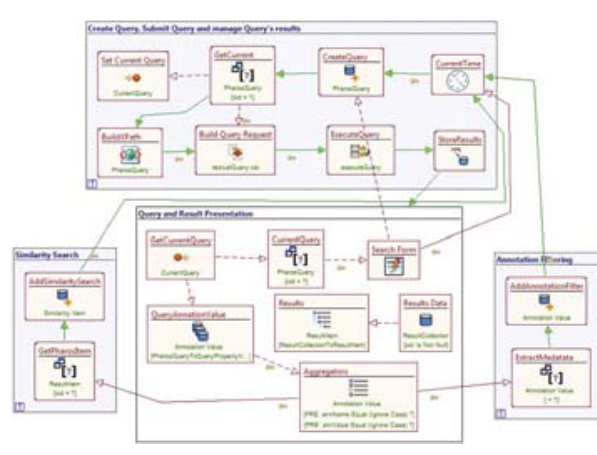

(a)

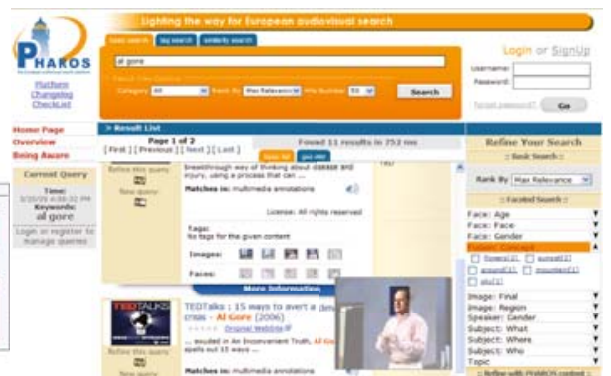

(b)

Fig. 2. (a) Hypertext model excerpt for the PHAROS QRP process; (b) Rendition example of the generated QRP Web interface

design of the CP process, based on ad hoc workflow models for multimedia content provisioning; (iii) an in-depth tour of the generated Pharos demonstrator, comprising several usage scenarios such as keyword- and content-based search, similarity search, faceted search, social and personalized content management, multi-modal and multi-channel user interfaces, provision of new contents, and update on the $\mathrm{CP}$ process.

For conceptual modeling of the user interface level of the QRP we exploit the Web Modeling Language (WebML) 6], a visual Domain Specific Language for the specification of contents, business processes, hypertexts, web service interaction [3, and visual presentation (including rich interface modeling 1]) of a Web application, and WebRatid, a MDE tool that provides WebML design and code generation facilities.

The specification of the CP process is based on the BPMN notation 3 for business process design. We support a subset of the complete BPMN semantics (coarsely, the part that maps to BPEL), and we extend it with search-specific aspects.

\section{The QRP Process}

Starting from a high level specification of a query, the QRP process automatically generates MPQF4 expressions for (i) keyword-based queries, (ii) annotationbased queries and results' filtering, (iii) user-specified tags, and (iv) similaritybased search on audio, images and faces. Queries can be iteratively built as an arbitrary combination of such options. Example of expressible queries are: a) find all the videos related to tourism in Bavaria, b) find the videos talking about $\mathrm{Al}$ Gore and where the speaker is Al Gore himself, c) find all the video containing faces similar to the uploaded one.

\footnotetext{
${ }^{2}$ WebRatio, http://www.webratio.com

${ }^{3}$ Business Process Management Notation, http://www.bpmn.org

${ }^{4}$ MPEG Query Format, http://www.mpqf.org
} 
The QRP process demo application offers two user interfaces: one for desktop $\mathrm{PC}$ and one for mobile terminal. The application performs query building, query execution on the PHAROS back-end, and rendering of the results. It also supports aspects like user-generated tags, queries storage, reuse and monitoring, in order to receive notifications (SMS or email) when new contents matching the given query are published.

Figure 2 shows an excerpt of the hypertext conceptual model designed for the PHAROS showcase, and a sample rendition of the user interface.

\section{The CP Process}

To allow easy and effective definition of the CP process, the BPMN notation is extended with search specific information to enable a more precise model transformation towards the running code. Every BPMN activity is associated with a type, describing the semantics of its behaviour, and a set of properties that describe the execution details (e.g., the service to be invoked and the parameters to be passed on). Examples of activity types : Retrieval (R), Transformation (T), Analysis (ANA), and Indexing (IDX) of content. An activity has a (possibly empty) set of input parameters and output parameters. The output flow of an activity can be associated to a guard condition, which is an OCL Boolean expression over the values of the output parameters. Figure 3 shows a simplified example of CP Process specified according to our extended notation. The design of the models is supported by a visual design tool and the CP process models configure a simple workflow engine that processes new contents.

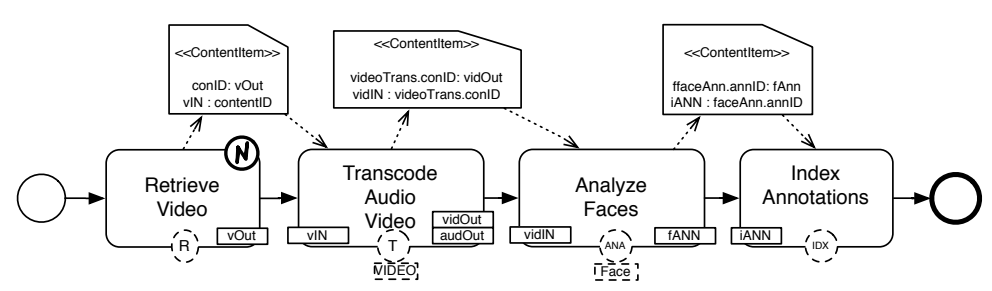

Fig. 3. Process model example for the Content Provisioning

\section{References}

1. Bozzon, A., et al.: Conceptual modeling and code generation for Rich Internet Applications. In: ICWE 2006, pp. 353-360 (2006)

2. Bates, M.: Toward an integrated model of information seeking and searching. The New Review of Information Behaviour Research 3, 1-15 (2002)

3. Brambilla, M., Ceri, S., Fraternali, P., Manolescu, I.: Process modeling in web applications. ACM TOSEM 15(4), 360-409 (2006) 
4. Debald, S., Nejdl, W., Nucci, F., Paiu, R., Plu, M.: Pharos, platform for search of audiovisual resources across online spaces. In: SAMT 2006 (2006)

5. Koch, N., Kraus, A., Cachero, C., Meliá, S.: Integration of business processes in web application models. J. Web Eng. 3(1), 22-49 (2004)

6. Ceri, S., Fraternali, P., Bongio, A., Brambilla, M., Comai, S., Matera, M.: Designing Data-Intensive Web Applications. Morgan Kaufmann, San Francisco (2002)

7. Torres, V., Pelechano, V.: Building business process driven web applications. In: Dustdar, S., Fiadeiro, J.L., Sheth, A.P. (eds.) BPM 2006. LNCS, vol. 4102, pp. 322-337. Springer, Heidelberg (2006) 